Bull. Korean Math. Soc. 48 (2011), No. 6, pp. 1207-1218

http://dx.doi.org/10.4134/BKMS.2011.48.6.1207

\title{
INTEGRAL DOMAINS WITH A FREE SEMIGROUP OF *-INVERTIBLE INTEGRAL $*$-IDEALS
}

\author{
Gyu Whan Chang ${ }^{\dagger}$ AND Hwankoo KIM ${ }^{\ddagger}$
}

\begin{abstract}
Let $*$ be a star-operation on an integral domain $R$, and let $\mathscr{I}_{*}^{+}(R)$ be the semigroup of $*$-invertible integral $*$-ideals of $R$. In this article, we introduce the concept of a $*$-coatom, and we then characterize when $\mathscr{I}_{*}^{+}(R)$ is a free semigroup with a set of free generators consisting of *-coatoms. In particular, we show that $\mathscr{I}_{*}^{+}(R)$ is a free semigroup if and only if $R$ is a Krull domain and each $v$-invertible $v$-ideal is $*$-invertible. As a corollary, we obtain some characterizations of $*$-Dedekind domains.
\end{abstract}

\section{Introduction}

Let $R$ be an integral domain, and let $*$ be a star-operation (defined later) on $R$. Let $\mathscr{F}_{*}(R)$ (resp., $\left.\mathscr{I}_{*}(R), \mathscr{P}(R)\right)$ be the set of nonzero fractional *ideals (resp., *-invertible $*$-ideals, principal ideals) of $R$. Then $\mathscr{F}_{*}(R)$ forms a commutative monoid under $*$-multiplication, that is, for any $A, B \in \mathscr{F}_{*}(R)$, $A * B:=(A B)_{*}$. Moreover $\mathscr{I}_{*}(R)$ is a subgroup of $\mathscr{F}_{*}(R)$ and $\mathscr{P}(R)$ is a subgroup of $\mathscr{I}_{*}(R)$. Let $\mathscr{F}_{*}^{+}(R)$ (resp., $\mathscr{I}_{*}^{+}(R), \mathscr{P}^{+}(R)$ ) be the positive cone of $\mathscr{F}_{*}(R)$ (resp., $\mathscr{I}_{*}(R), \mathscr{P}(R)$ ) which consists of the nonzero integral $*$-ideals (resp., $*$-invertible $*$-ideals, principal ideals) of $R$. The structure of an integral domain $R$ depends heavily on the properties of $\mathscr{F}_{*}(R), \mathscr{F}_{*}^{+}(R), \mathscr{P}(R)$, or $\mathscr{P}^{+}(R)$. For instance, it is well-known that $R$ is a Dedekind domain if and only if $\mathscr{F}_{d}^{+}(R)$ is a free semigroup with base $\operatorname{Spec}(R) \backslash\{0\}$. In [6], it was determined when $\mathscr{F}_{d}(R)$ is finitely generated as a monoid. Recently in [4], it was further investigated and extended to a commutative ring with zero divisors. In [8], among other things, it was shown that $R$ is a Krull domain if and only if $\mathscr{F}_{t}^{+}(R)$ is a free semigroup with a set of free generators consisting of $t$-nonfactorable ideals. Later the concept of $*$-nonfactorability of ideals was further studied in

Received July 4, 2010; Revised July 30, 2011.

2010 Mathematics Subject Classification. 13A15, 13F15, $13 \mathrm{E} 99$.

Key words and phrases. star-operation, free semigroup of $*$-invertible $*$-ideals, $*$-locally factorial Krull domain, $\pi$-domain, $*$-Dedekind domain.

† This work was supported by the University of Incheon Research Grant in 2010.

$\ddagger$ This research was supported by Basic Science Research Program through the National Research Foundation of Korea(NRF) funded by the Ministry of Education, Science and Technology(2010-0011996). 
$[10,18,19]$. In particular, it was shown in [10, Theorem 3.2] that if $\mathscr{F}_{*}^{+}(R)$ is a free semigroup with a set of free generators consisting of *-nonfactorable ideals, then $R$ is a Krull domain. Finally, in [20], it was characterized when $\mathscr{I}_{d}^{+}(R)$ is a free semigroup with a system of generators consisting of coatoms.

In this article, we define the notion of $*$-coatoms for any star-operation $*$ and we then characterize when $\mathscr{I}_{*}^{+}(R)$ is a free semigroup with a set of free generators consisting of $*$-coatoms. More precisely, we show that $\mathscr{I}_{*}(R)$ is a free semigroup if and only if $R$ is a Krull domain and $\mathscr{I}_{v}(R)=\mathscr{I}_{*}(R)$ if and only if every nonzero principal ideal of $R$ can be expressed as a finite *-product of height-one prime ideals. In particular, if $*$ is of finite character, then $\mathscr{I}_{*}(R)$ is a free semigroup if and only if each nonzero $*$-locally principal ideal of $R$ is *-invertible and $R_{M}$ is a factorial domain for all $*$-maximal ideals $M$ of $R$. As a byproduct, we obtain some characterizations of $*$-Dedekind domains.

Let $R$ be an integral domain with quotient field $K$. Let $\mathscr{F}(R)$ be the set of nonzero fractional ideals of $R$. A mapping $A \mapsto A_{*}$ of $\mathscr{F}(R)$ into $\mathscr{F}(R)$ is called a star-operation on $R$ if the following conditions are satisfied for all $a \in K \backslash\{0\}$ and $A, B \in \mathscr{F}(R)$ :

(i) $(a R)_{*}=a R,(a A)_{*}=a A_{*}$;

(ii) $A \subseteq A_{*}$, if $A \subseteq B$, then $A_{*} \subseteq B_{*}$;

(iii) $\left(A_{*}\right)_{*}=A_{*}$.

It is easy to show that for all $A, B \in \mathscr{F}(R),(A B)_{*}=\left(A B_{*}\right)_{*}=\left(A_{*} B_{*}\right)_{*}$. An $A \in \mathscr{F}(R)$ is called a $*$-ideal if $A=A_{*}$. A $*$-ideal is called a $*$-maximal ideal if it is maximal among proper integral $*$-ideals. We denote by $*-\operatorname{Max}(R)$ (resp., $*$-Spec $(R))$ the set of all *-maximal ideals (resp., prime $*$-ideals) of $R$.

Given any star-operation $*$ on $R$, we can construct another star-operation $*_{f}$ defined by $A_{*_{f}}:=\bigcup\left\{J_{*} \mid J\right.$ is a nonzero finitely generated subideal of $\left.A\right\}$ for $A \in \mathscr{F}(R)$. Clearly, if $A \in \mathscr{F}(R)$ is finitely generated, then $A_{*}=A_{*_{f}}$. We say that $*$ is of finite character if $*=*_{f}$ and that $*_{f}$ is the finite character star-operation induced by $*$. It is well-known that if $*=*_{f}$, then $*-\operatorname{Max}(R) \neq \emptyset$ when $R$ is not a field; a $*$-maximal ideal is a prime ideal; each proper integral $*-$ ideal is contained in a $*$-maximal ideal; each prime ideal minimal over a $*$-ideal is a prime $*$-ideal (in particular, each height-one prime ideal is a prime $*$-ideal); and $R=\bigcap_{P \in *-\operatorname{Max}(R)} R_{P}$. Let $*$ be any star-operation on $R$. An $A \in \mathscr{F}(R)$ is said to be $*$-invertible if $\left(A A^{-1}\right)_{*}=R$, where $A^{-1}=\{x \in K \mid x A \subseteq R\}$. We say that $A \in \mathscr{F}(R)$ is of $*$-finite type if $A_{*}=B_{*}$ for some finitely generated ideal $B$ of $R$. Also, $A \in \mathscr{F}(R)$ is said to be $*$-locally principal if $A R_{P}$ is principal for all $*$-maximal ideals $P$ of $R$. It is well-known that $A$ is $*_{f}$-invertible if and only if $A$ is of $*_{f}$-finite type and $A$ is $*_{f}$-locally principal.

The most important examples of star-operations are (1) the $d$-operation defined by $A_{d}:=A,(2)$ the $v$-operation defined by $A_{v}:=\left(A^{-1}\right)^{-1}$, (3) the $t$-operation defined by $t:=v_{f}$, and (4) the $w$-operation defined by $A_{w}:=$ $\left\{x \in K \mid J x \subseteq A\right.$ for some finitely generated ideal $J$ with $\left.J^{-1}=R\right\}$ for $A \in \mathscr{F}(R)$. Note that all star-operations above except for $v$ are of finite 
character. For any star-operation $*$ on $R$ and for any $A \in \mathscr{F}(R)$, we have that $A \subseteq A_{*} \subseteq A_{v}$ (i.e., $\left.d \leq * \leq v\right)$ and $A \subseteq A_{*_{f}} \subseteq A_{t}$; so $\left(A_{*}\right)_{v}=A_{v}=\left(A_{v}\right)_{*}$ and $\left(A_{*_{f}}\right)_{t}=A_{t}=\left(A_{t}\right)_{*_{f}}$. In particular, a $v$-ideal (resp., $t$-ideal) is a $*$-ideal (resp., $*_{f}$-ideal). General references for any undefined terminology or notation are $[15,16]$.

\section{When $\mathscr{I}_{*}^{+}(R)$ is a free semigroup}

Throughout this section, $R$ denotes an integral domain with quotient field $K, *$ is a star-operation on $R, *_{f}$ is the finite character star-operation on $R$ induced by $*$, and $\mathscr{I}_{*}^{+}(R)$ is the semigroup of $*$-invertible integral $*$-ideals of $R$. In this section, we study when $\mathscr{I}_{*}^{+}(R)$ is a free semigroup.

As mentioned in the introduction, in [8], the authors introduced the concepts of $*$-nonfactorable ideals and (unique) $*$-factorable domains, and they then characterized several integral domains including Krull domains using these concepts. We say that an ideal $N$ of $R$ is $*$-nonfactorable if it is a proper *ideal and $N=(A B)_{*}$, where $A$ and $B$ are ideals of $R$, implies either $A_{*}=R$ or $B_{*}=R$. We also say that an integral domain $R$ is a $*$-factorable domain (resp., unique *-factorable domain) if every proper $*$-ideal of $R$ can be factored (resp., factored uniquely) into a $*$-product of $*$-nonfactorable ideals.

Definition. An ideal $A$ of $\mathscr{I}_{*}^{+}(R)$ is called a $*$-coatom of $\mathscr{I}_{*}^{+}(R)$ if $A$ is not expressible as a nontrivial $*$-product of $*$-invertible ideals of $R$.

We remark that the $*$-coatoms of $\mathscr{I}_{*}^{+}(R)$ coincide with the maximal elements of $\mathscr{I}_{*}^{+}(R)$ (with respect to set inclusion). By definition a $*$-coatom is a $*$ invertible $*$-ideal. Hence it is precisely a $*$-nonfactorable $*$-invertible $*$-ideal. We next recall some results from [21, Theorem 1.1] on $*$-invertible ideals, which are very useful in the subsequent arguments.

Lemma 2.1. If $I$ is a nonzero fractional ideal of $R$, then

(1) $I_{*_{f}} \subseteq I_{t}$;

(2) If $I$ is *-invertible, then $I$ is $v$-invertible and $I_{*}=I_{v}$;

(3) If $I$ is $*_{f}$-invertible, then $I$ is t-invertible and $I_{*}=I_{*_{f}}=I_{t}=I_{v}$.

Lemma 2.2. If $R$ satisfies ACC on *-invertible *-ideals, then every $*$-invertible *-ideal is expressible as a (finite) *-product of *-coatoms.

Proof. This follows from the following easy observation: Let $I \subseteq J$ be $*$-ideals of $R$ with $J, *$-invertible. Then there exists a $*$-ideal $A$ such that $I=(J A)_{*}$.

The proof of the following lemma is essentially the same as that of $[8$, Lemma 11]. However for the sake of completeness we include its proof.

Lemma 2.3. Suppose that $\mathscr{I}_{*}^{+}(R)$ is a free semigroup with a set of free generators consisting of $*$-coatoms, and let $N$ be a proper $*$-invertible $*$-ideal of $R$. Then $N$ is a *-coatom if and only if $N$ is prime (if and only if $N$ is *-nonfactorable). 
Proof. Let $N$ be a $*$-coatom. Let $a b \in N$ for nonzero nonunits $a, b \in R$ with $a \notin N$. Then $a R=\left(P_{1} \cdots P_{r}\right)_{*}$, where each $P_{i}$ is a $*$-coatom. Note that each $P_{i} \neq N$ since $a \notin N$. Also, $b R=\left(Q_{1} \cdots Q_{s}\right)_{*}$, where each $Q_{j}$ is a $*$-coatom. Note that $a b N^{-1}$ is a proper $*$-invertible $*$-ideal (if $a b N^{-1}=R$, then $N=\left(\left(a b N^{-1}\right) N\right)_{*}=a b R=(a R)(b R)$ is not a $*$-coatom); so we may write $a b N^{-1}=\left(M_{1} \cdots M_{u}\right)_{*}$, where each $M_{k}$ is a $*$-coatom. Thus $a b R=$ $\left(N M_{1} \cdots M_{u}\right)_{*}=\left(P_{1} \cdots P_{r} Q_{1} \cdots Q_{s}\right)_{*}$. Since $\mathscr{I}_{*}^{+}(R)$ is a free semigroup with a set of free generators consisting of $*$-coatoms, we have that $N=Q_{j}$ for some $j$. Thus $b \in b R=\left(Q_{1} \cdots Q_{s}\right)_{*} \subseteq N$, and hence $N$ is prime. Conversely, it was already observed in [8] that a $*$-invertible prime $*$-ideal is $*$-nonfactorable, and hence a $*$-coatom.

Lemma 2.4. If every nonzero proper principal ideal of $R$ decomposes into a *-product of prime *-ideals, then the set of the height-one primes equals the set of the $*$-invertible prime $*$-ideals.

Proof. Let $P$ be a $*$-invertible prime $*$-ideal of $R$ and assume that the height of $P$ is greater than 1 . Then there exists a nonzero prime ideal $Q \subsetneq P$. For $0 \neq q \in Q$, we have $Q \supseteq q R=\left(P_{1} \cdots P_{s}\right)_{*}$, where the $P_{i}$ are prime $*$-ideals. It then follows that $P \supsetneq Q \supseteq P_{i}$ for some $i(1 \leq i \leq s)$. Thus $P_{i}=(P Z)_{*}$ for some $*$-invertible $*$-ideal $Z \neq R$. This implies $Z \subseteq P_{i}$. So if we replace $Z$ by $P_{i}$ in the equality $P_{i}=(P Z)_{*}$ and apply the $*$-invertibility of $P_{i}$, we get a contradiction. For the reverse inclusion, let $P$ be a height-one prime ideal of $R$. Then $P$ is minimal over a nonzero principal ideal $a R=\left(P_{1} \cdots P_{n}\right)_{*}$, where the $P_{i}$ are prime $*$-ideals. Thus $P=P_{i}$ for some $i$, and so $P$ is a $*$-invertible prime $*$-ideal.

Let $X^{1}(R)$ be the set of height-one prime ideals of $R$. An integral domain $R$ is a Krull domain if (i) $R_{P}$ is a rank-one DVR for each $P \in X^{1}(R)$, (ii) $R=\bigcap_{P \in X^{1}(R)} R_{P}$, and (iii) each nonzero element of $R$ is contained in finitely many height-one prime ideals of $R$. It is well known that $R$ is a Krull domain if and only if each nonzero proper principal ideal of $R$ is a $t$-product of $(t$ invertible) prime ideals [17, Theorem 3.9].

We say that $R$ is a $*$-Schreier domain if $\mathscr{I}_{*}(R)$ is a Riesz group. More precisely, $R$ is a $*$-Schreier domain if whenever $A, B_{1}, B_{2}$ are $*$-invertible $*$ ideals of $R$ and $A \supseteq B_{1} B_{2}$, then $A=\left(A_{1} A_{2}\right)_{*}$ for some (*-invertible) *-ideals $A_{1}, A_{2}$ of $R$ with $A_{i} \supseteq B_{i}$ for $i=1,2$. We remark that the concepts of $t$-Schreier domains and $d$-Schreier domains (as the name of quasi-Schreier domains) were already introduced as a generalization of Prüfer $v$-multiplication domains and proved very useful in $[5,11,12]$. An integral domain $R$ is called a $*-G C D$ domain if the intersection of two $*$-invertible $*$-ideal is $*$-invertible (cf., $[16$, Definition 17.6]). Then $d$-GCD domains are exactly generalized GCD-domains, which are introduced in [1] and further investigated in [3]. For $I, J \in \mathscr{I}_{*}(R)$, an ordered group under the partial order $A \leq B \Leftrightarrow B \subseteq A, \sup (I, J)$ exists if and only if $I \cap J$ is *-invertible, and hence $\sup (I, J)=I \cap J$. It follows that 
$R$ is a $*$-GCD domain if and only if $\mathscr{I}_{*}(R)$ is a lattice-ordered group (cf., [3, Theorem 1]). Thus every $*$-GCD domain is a $*$-Schreier domain.

Theorem 2.5. The following conditions are equivalent for an integral domain $R$.

(1) $\mathscr{I}_{*}^{+}(R)$ is a free semigroup with a set of free generators consisting of *-coatoms.

(2) $R$ satisfies ACC on *-invertible *-ideals and $R$ is a*-GCD domain.

(3) $R$ satisfies ACC on *-invertible *-ideals and $R$ is a*-Schreier domain.

(4) $R$ satisfies $A C C$ on $*$-invertible $*$-ideals and the $*$-coatoms are prime *-ideals.

(5) $R$ is a Krull domain and $\mathscr{F}_{v}(R)=\mathscr{I}_{*}(R)$.

(6) $R$ is a Krull domain and $\mathscr{F}_{v}^{+}(R)=\mathscr{I}_{*}^{+}(R)$.

(7) $R$ satisfies ACC on *-invertible *-ideals and every nonzero principal ideal of $R$ can be uniquely decomposed into a $*$-product of $*$-coatoms.

(8) Every nonzero principal ideal of $R$ uniquely decomposes into a $*$-product of prime $*$-ideals.

(9) Every nonzero principal ideal of $R$ can be written as a finite *-product of height-one prime ideals.

Proof. $(1) \Rightarrow(2)$. Note that the ordered group $\mathscr{I}_{*}(R)$ is isomorphic to a direct sum of copies of $\mathbb{Z}$, the additive group of integers. Thus by the remark before Theorem $2.5 R$ is a $*$-GCD domain. The rest is clear.

$(2) \Rightarrow(3)$. This follows from the remark before Theorem 2.5.

$(3) \Rightarrow(4)$. It is easily seen that in a $*$-Schreier domain every $*$-coatom is a prime $*$-ideal.

$(4) \Rightarrow(1)$. The existence of a decomposition into $*$-coatoms follows from Lemma 2.2. From the primeness of the $*$-coatoms, it follows that they are free generators; that is, the equality $\left(P_{1} \cdots P_{m}\right)_{*}=\left(Q_{1} \cdots Q_{s}\right)_{*}$ implies that $m=s$ and that there exists a permutation $\sigma$ such that $P_{i}=Q_{\sigma(i)}$.

$(1) \Rightarrow(7)$. This is clear.

$(7) \Rightarrow(4)$. Let $P$ be a $*$-coatom of $\mathscr{I}_{*}^{+}(R)$, and let $a, b$ be nonzero nonunits of $R$ with $a b \in P$. It suffices to show that $a \in P$ or $b \in P$. By (7), there are some $*$-coatoms $P_{1}, \ldots, P_{n}, Q_{1}, \ldots, Q_{k}$ such that $a R=\left(P_{1} \cdots P_{n}\right)_{*}$ and $b R=\left(Q_{1} \cdots Q_{k}\right)_{*} ;$ in particular, $a b R=\left(P_{1} \cdots P_{n} Q_{1} \cdots Q_{k}\right)_{*}$. Since $a b R \subseteq P$, we have $I:=a b P^{-1} \subseteq R$ and $a b R=(P I)_{*}$. Clearly, $I$ is $*$-invertible, and hence $I=\left(P_{1}^{\prime} \cdots P_{l}^{\prime}\right)_{*}$ for some $*$-coatoms $P_{i}^{\prime}$; so $\left(P_{1} \cdots P_{n} Q_{1} \cdots Q_{k}\right)_{*}=a b R=$ $\left(P P_{1}^{\prime} \cdots P_{l}^{\prime}\right)_{*}$. Hence by the uniqueness, we have $P=P_{i}$ or $P=Q_{j}$, and therefore $a \in P$ or $b \in P$.

$(5) \Leftrightarrow(6) \Rightarrow(4)$. These are clear because each $v$-ideal is a $*$-ideal.

$(7) \Rightarrow(5)$. We first note that each nonzero nonunit of $R$ can be written as a finite $*$-product of height-one prime $*$-ideals of $R$ by Lemma 2.4 and the $(7)$ $\Rightarrow(4)$ above. Next, let $P$ be a height-one prime ideal of $R$. Then by Lemma 2.4, $P$ is a $*$-invertible prime $*$-ideal. Thus we have that $P \subsetneq P P^{-1}$; so $R_{P}$ is a rank-one DVR. Also, $a R=\left(P_{1} \cdots P_{n}\right)_{*}$ implies that $a$ is contained in a 
finite number of height-one prime ideals of $R$. Suppose that $a R: b R \subsetneq R$ for $a, b \in R$. Then the proof of [10, Theorem 3.2] also shows that $a R: b R \subseteq P$ for some $P \in X^{1}(R)$, and thus $R=\bigcap_{P \in X^{1}(R)} R_{P}$ [15, Ex. 22, p. 52]. Thus $R$ is a Krull domain.

Next, let $I$ be a $v$-ideal of $R$. Then $I=\left(P_{1} \cdots P_{n}\right)_{v}$ for some height-one prime ideals $P_{1}, \ldots, P_{n}$ of $R$ because $R$ is a Krull domain. Note that each $P_{i}$ is $*$-invertible by the above proof; so $P_{1} \cdots P_{n}$ is $*$-invertible, and hence by Lemma $2.1 I=\left(P_{1} \cdots P_{n}\right)_{*}$. Since $I^{-1}=\left(P^{-1} \cdots P_{n}^{-1}\right)_{*}$, we have $\left(I I^{-1}\right)_{*}$ $=\left(\left(P_{1} \cdots P_{n}\right)_{*} P^{-1} \cdots P_{n}^{-1}\right)_{*}=\left(\left(P_{1} P_{1}^{-1}\right)_{*} \cdots\left(P_{n} P_{n}^{-1}\right)_{*}\right)_{*}=R$. Thus $I$ is $*-$ invertible.

$(1) \Rightarrow(8)$. This follows from Lemma 2.3 .

$(8) \Rightarrow(9)$. This follows from Lemma 2.4 .

$(9) \Rightarrow(5)$. Note that by $(9)$ each nonzero $a \in K$ can be uniquely written as $a R=\left(\prod\left\{P^{v_{P}(a)} \mid P \in X^{1}(R)\right\}\right)_{*}$, where $v_{P}(a) \in \mathbb{Z}$ and $v_{P}(a)=0$ for almost all $P$. Hence for each height-one prime $P$ the assignment $v_{P}(a)$ defines a discrete valuation on $K$. Since $a \in R$ if and only if $v_{P}(a) \geq 0$ for all $P$, denoting by $V_{P}$ the valuation ring of $v_{P}$, we have that $R=\bigcap V_{P}$ with finite character. Thus $R$ is a Krull domain such that each $v$-ideal is $*$-invertible.

We remark that for $*=d$, Theorem 2.5 is essentially in $[11,14,20]$.

\section{When $\mathscr{I}_{*}^{+}(R)$ is a free semigroup for $*=*_{f}$}

As in Section 2, we denote by $R$ an integral domain with quotient field $K, *$ is a star-operation on $R, *_{f}$ is the finite character star-operation on $R$ induced by $*$, and $\mathscr{I}_{*}^{+}(R)$ is the semigroup of $*$-invertible integral $*$-ideals of $R$. In this section, we study when $\mathscr{I}_{*}^{+}(R)$ is a free semigroup for $*=*_{f}$.

We first give an example of a star-operation $*$ such that $*$ is not of finite type, yet Theorem 2.5 holds for it. Following the referee's remark, such a staroperation has the potential of being very useful for answering other questions in the literature.

Example 3.1. Let $D$ be a $\pi$-domain, $\left\{X_{\alpha}\right\}$ an infinite set of indeterminates over $D$, and $\left\{Q_{\lambda}\right\}$ the set of (nonzero) finitely generated prime ideals of $D\left[\left\{X_{\alpha}\right\}\right]$. It is known that $D\left[\left\{X_{\alpha}\right\}\right]$ is a $\pi$-domain (cf. $[1$, p. 200]) and $D\left[\left\{X_{\alpha}\right\}\right]=\bigcap_{\lambda} D\left[\left\{X_{\alpha}\right\}\right]_{Q_{\lambda}}$. For each nonzero fractional ideal $A$ of $D\left[\left\{X_{\alpha}\right\}\right]$, if we define

$$
A_{*}=\bigcap_{\lambda} A D\left[\left\{X_{\alpha}\right\}\right]_{Q_{\lambda}},
$$

then $*$ is a star-operation on $D\left[\left\{X_{\alpha}\right\}\right][2$, Theorem 1]. Again by [2, Theorem $1]$, if we let $M=\left(\left\{X_{\alpha}\right\}\right)$, then $M_{*}=D\left[\left\{X_{\alpha}\right\}\right]$, because $M$ is not contained in any of $Q_{\lambda}$. But $M_{*_{f}} \neq D\left[\left\{X_{\alpha}\right\}\right]$ by the definition of $*$. Thus $* \neq *_{f}$, i.e., * is not of finite type. Finally, since $D\left[\left\{X_{\alpha}\right\}\right]$ is a $\pi$-domain, each nonzero principal ideal of $D\left[\left\{X_{\alpha}\right\}\right]$ is a finite product (and thus $*$-product since $d<*$ ) 
of height-one prime ideals. Thus * is not of finite type but Theorem 2.5 holds for this star-operation.

The following result comes from Lemma 2.1 and [21, Corollary 4.5].

Lemma 3.2. If $P$ is a $*_{f}$-invertible prime ideal of $R$ such that $P_{*_{f}} \subsetneq R$, then

(a) If $I$ is $a *_{f}$-invertible $*_{f}$-ideal containing $P$, then $I=P=P_{*_{f}}$;

(b) $P$ is a t-invertible t-maximal ideal.

To characterize *-locally factorial Krull domains in terms of "*-LPI domain" in Theorem 3.7, we need the notion of a $*-L P I$ domain, that is, an integral domain in which every nonzero $*$-locally principal ideal is $*$-invertible. This notion first appeared in [22] for $*=t$, in the context of integral domains, essentially Prüfer $v$-multiplication domains and slightly more general domains. Then it appears in [12] in the context of $t$-Schreier domains and in [7] as a defining property for "Locally Principal ideals are Invertible"-domains with $*=d$.

Lemma 3.3. Let $*$ be of finite character on the integral domain $R$, and suppose that $R$ is a*-LPI domain. If $R_{M}$ satisfies ACC on principal ideals of $R_{M}$ for every *-maximal ideal $M$ of $R$, then $R$ satisfies $A C C$ on *-invertible *-ideals of $R$.

Proof. Consider an increasing chain of $*$-invertible $*$-ideals: $I_{1} \subseteq I_{2} \subseteq \cdots$. Let $I:=\lim I_{n}$. Then $I$ is a $*$-ideal of $R$, since $*$ is of finite character. It is sufficient to show that $I$ is $*$-invertible.

For every *-maximal ideal $M$, we have $I R_{M}=I \otimes_{R} R_{M}=\lim _{\rightarrow}\left(I_{n} \otimes_{R} R_{M}\right)=$ $\lim _{\rightarrow}\left(I_{n} R_{M}\right)=\lim _{\rightarrow}\left(a_{n} R_{M}\right)=a R_{M}$, where all $a_{n}$ and $a$ are nonzero elements of $R_{M}$. It then follows that $I$ is $*$-invertible, since $I$ is a $*$-locally principal ideal.

Lemma 3.4 (cf. [13, Lemma 4.23]). If $P$ is a $*$-invertible prime $*$-ideal and $Q$ is a *-invertible *-ideal such that $Q \nsubseteq P$, then $\left(P^{k} \cap Q\right)_{*}=\left(P^{k} Q\right)_{*}$ for all integers $k>0$.

Proof. We have $\left(P^{k} \cap Q\right)_{*}=\left(P^{k} A\right)_{*}=(Q B)_{*}$ for some $*$-ideals $A$ and $B$, and thus $(Q B)_{*} \subseteq\left(P^{k}\right)_{*}$. Since $Q \nsubseteq P$, from [16, Theorem 13.2(iv)] we deduce that $B \subseteq\left(P^{k}\right)_{*}$. Thus we have $B=\left(P^{k} C\right)_{*}$ for some $*$-ideal $C$, and hence $\left(P^{k} \cap Q\right)_{*}=(Q B)_{*}=\left(P^{k} Q C\right)_{*} \subseteq\left(P^{k} Q\right)_{*}$. And the reverse inclusion is obvious.

Proposition 3.5 (cf. [13, Corollary 4.24]). If $P_{i}(1 \leq i \leq k)$ are $*$-invertible prime *-ideals of height-one such that $P_{i} \neq P_{j}$ for $i \neq j$, then $\left(\bigcap_{1}^{k} P_{i}^{n_{i}}\right)_{*}=$ $\left(\prod_{1}^{k} P_{i}^{n_{i}}\right)_{*}$.

Proof. We proceed by induction on the number $k$ of ideals. For $k=1$ the assertion is obvious. Assume that it is true for $k=s$, and deduce it for $k=s+1$. We need to verify that $\left(\left(\bigcap_{1}^{s} P_{i}^{n_{i}}\right) \cap P_{s+1}^{n_{s+1}}\right)_{*}=\left(\left(\prod_{1}^{s} P_{i}^{n_{i}}\right) \cdot P_{s+1}^{n_{s+1}}\right)_{*}$. 
By induction hypothesis, $\left(\bigcap_{1}^{s} P_{i}^{n_{i}}\right)_{*}=\left(\prod_{1}^{s} P_{i}^{n_{i}}\right)_{*}$. Now the assertion follows from Lemma 3.4.

Lemma 3.6. Let $*$ be of finite character on the integral domain $R$, and suppose that $R$ is a*-LPI domain. If $R_{M}$ is factorial for each $*$-maximal ideal $M$, then every nonzero principal ideal of $R$ decomposes into a*-product of prime *ideals.

Proof. It follows from Lemma 3.3 that $R$ satisfies ACC on $*$-invertible $*$-ideals of $R$, and hence every nonzero proper principal ideal $a R$ of $R$ decomposes into a *-product of $*$-coatoms, say $a R=\left(\prod_{i=1}^{s} P_{i}^{n_{i}}\right)_{*}$ (all the $P_{i}$ are distinct). We will prove that $\left\{P_{i}\right\}_{1 \leq i \leq s}$ is the set of height-one prime ideals containing $a$, and $a \in\left(P_{i}^{n_{i}}\right)_{*}$ and $a \notin\left(P_{i}^{n_{i}+1}\right)_{*}$.

Consider the set $X_{a}^{(1)}$ of height-one prime ideals of $R$ containing $a$.

(1) $X_{a}^{(1)}$ is not empty. Indeed, from the fact that $\frac{a}{1} \in Q$ for some heightone prime ideal $Q$ of $R_{M}$ (where $M$ is a $*$-maximal ideal of $R$ ) it follows that $a \in Q \cap R \in X_{a}^{(1)}$.

(2) Every element $P$ of $X_{a}^{(1)}$ is *-invertible. Indeed, let $M$ be an arbitrary *-maximal ideal of $R$. Then $P R_{M}$ is a height-one prime ideal of $R_{M}$ if $P \subseteq M$, and so a $t$-ideal. Since $R_{M}$ is factorial, $P R_{M}$ is principal by [17, p. 284], and hence $P$ is $*$-locally principal. Thus $P$ is $*$-invertible.

(3) $X_{a}^{(1)}$ is finite. Indeed, if $P \in X_{a}^{(1)}$, then $\left(P_{1}^{n_{1}} \cdots P_{s}^{n_{s}}\right)_{*}=a R \subseteq P$. Thus we have $P_{i} \subseteq P$. Since $P$ is a $*$-invertible $*$-ideal and $P_{i}$ is a $*$-coatom, $P=P_{i}$. Thus $X_{a}^{(1)}$ is finite.

(4) Let $X_{a}^{(1)}=\left\{Q_{j}\right\}_{j \in J}$, and also $a \in\left(Q_{j}^{m_{j}}\right)_{*}$ and $a \notin\left(Q_{j}^{m_{j}+1}\right)_{*}$. By Proposition 3.5, we have $\left(\prod_{j \in J} Q_{j}^{m_{j}}\right)_{*}=\left(\bigcap_{j \in J} Q_{j}^{m_{j}}\right)_{*} \supseteq\left(\prod_{i}^{s} P_{i}^{n_{i}}\right)_{*}$. It remains to show that for every $P_{i}$ there exists $Q_{j(i)} \in X_{a}^{(1)}$ such that $Q_{j(i)}=P_{i}$. Let $M$ be a *-maximal ideal of $R$ such that $a R \subseteq P_{i} \subseteq M$. In $R_{M}$ there exists a minimal prime ideal $N \supseteq P_{i} R_{M}$. We have that $a \in P_{i} \subseteq N \cap R$ and $N \cap R$ is a height-one prime ideal in $R$, and thus it is $*$-invertible. From this we deduce that $N \cap R=Q_{j(i)} \in X_{a}^{(1)}$. The assertion is thus proved, since $Q_{j(i)}=P_{i}$.

Let $V$ be a non-discrete valuation domain of (Krull) dimension 1 . Then $V$ does not have a $v$-maximal ideal, and hence $V_{M}$ is a factorial domain for each $v$-maximal ideal $M$ of $V$. However, each nonzero principal ideal of $V$ cannot be expressed as a finite $v$-product of $v$-maximal ideals. Thus, in Lemma 3.6, we need the assumption that $*$ is of finite character.

In the following, we characterize *-locally factorial Krull domains.

Theorem 3.7. If $*$ is of finite character, then the statements in Theorem 2.5 are equivalent to each of the following:

(10) $R$ is a*-LPI domain and $R_{M}$ is factorial for every *-maximal ideal $M$ of $R$. 
(11) $R$ is a Krull domain and $R_{M}$ is factorial for every $*$-maximal ideal $M$ of $R$.

Proof. $(7) \Rightarrow(10)$. Let $I$ be a nonzero *-locally principal ideal of $R$. Then $I$ is a $t$-ideal, and since $R$ is a Krull domain, $I$ is $t$-invertible. Therefore $I$ is *-invertible by (7). Next, let $M$ be a *-maximal ideal of $R$. Then $R_{M}$ is a Krull domain; so to show that $R_{M}$ is a factorial domain, it suffices to show that each height-one prime ideal of $R_{M}$ is principal. Let $Q$ be a height-one prime ideal of $R_{M}$. Then $Q=P R_{M}$ for some height-one prime ideal $P$ of $R$. By (7), $P$ is *-invertible, and hence $Q=P R_{M}$ is invertible. Thus $Q$ is principal because $R_{M}$ is quasi-local.

$(10) \Rightarrow(8)$. This follows from Lemma 3.3 and Lemma 3.6.

$(9) \Leftrightarrow(11)$. [16, Exercise 22.7].

We remark that if we take $*=d$ in Theorem 3.7 , then it is well known that the statements in Theorem 3.7 are equivalent to $R$ being a $\pi$-domain $([1,17])$. In the case of $*=t$ (resp., $w$ ), it follows from [17, Theorem 3.9] (resp., [18, Theorem 3.6]) that the equivalent conditions in Theorem 3.7 are equivalent to $R$ being a Krull domain.

Recall that $R$ is a $*$-Dedekind domain if every nonzero ideal of $R$ is *invertible, while $R$ is a $*$-Prüfer domain if every nonzero finitely generated of $R$ is $*$-invertible. Hence a Dedekind domain is a $d$-Dedekind domain; a Prüfer domain is a $d$-Prüfer domain; and a $v$-domain is a $v$-Prüfer domain. It is well known that each nonzero ideal of $R$ is $v$-invertible if and only if $R$ is completely integrally closed [15, Theorem 34.3]. Hence $R$ is a $v$-Dedekind domain if and only if $R$ is completely integrally closed.

Corollary 3.8. Let $*$ be a finite character star-operation on an integral domain $R$. Then the following conditions are equivalent.

(1) $R$ is a*-Dedekind domain.

(2) Every *-maximal ideal of $R$ is *-invertible, and $R$ satisfies $A C C$ on *-invertible $*$-ideals of $R$.

(3) $\mathscr{I}_{*}^{+}(R)$ is a free semigroup with a system of generators consisting of *-coatoms, and $(I+J)_{*}$ is *-invertible for any $I, J \in \mathscr{I}_{*}^{+}(R)$.

(4) $R$ is a *-Prüfer domain, and $\mathscr{I}_{*}^{+}(R)$ is a free semigroup with a system of generators consisting of $*$-coatoms.

(5) $R$ is a Krull domain and $*=t$.

(6) $R$ is a *-Prüfer domain and $R$ satisfies ACC on *-invertible integral $*$-ideals of $R$.

(7) $R$ is a*-Prüfer and *-factorable domain.

(8) $R$ is a unique *-factorable domain and every prime *-ideal of $R$ is *maximal.

Proof. $(1) \Rightarrow(4)$. Clearly, $R$ is a $*$-Prüfer domain. Also, $\mathscr{I}_{*}^{+}(R)$ is free with base $*-\operatorname{Spec}(R)=X^{1}(R)$ [16, Corollary 23.3 i)]. Now the second assertion 
follows from the fact that a $*$-invertible prime $*$-ideal is $*$-nonfactorable, and hence a $*$-coatom.

$(4) \Rightarrow(3)$. This follows because every $*$-finite type ideal of a $*$-Prüfer domain is $*$-invertible.

$(3) \Rightarrow(2)$. It suffices to show that every $*$-maximal ideal of $R$ is $*$-invertible. Let $M$ be a $*$-maximal ideal of $R$ and let $0 \neq a \in M$. Then by (3), $a R=$ $\left(P_{1}^{k_{1}} \ldots P_{n}^{k_{n}}\right)_{*}$, where $P_{1}, \ldots, P_{n}$ are $*$-coatoms with $P_{s} \neq P_{r}$ for $s \neq r$. Hence $P_{i} \subseteq M$ for some $i$. We will show that $M=P_{i}$. Suppose for a contradiction that there exists $b \in M$ but $b \notin P_{i}$. Then $b R=\left(Q_{1}^{m_{1}} \cdots Q_{l}^{m_{l}}\right)_{*} \subseteq M$, where $\left\{Q_{i}\right\}$ is a set of distinct *-coatoms of $R$. Hence $Q_{k} \subseteq M$ for some $k$. In addition, $Q_{k} \neq P_{i}$. Since $\left(Q_{k}+P_{i}\right)_{*}$ is a $*$-invertible $*$-ideal by $(3),\left(Q_{k}+P_{i}\right)_{*}$ is a $t$-invertible $t$-ideal of $R$. But then $M \supseteq\left(Q_{k}+P_{i}\right)_{*}=\left(Q_{k}+P_{i}\right)_{t}=R$, since $Q_{k}$ and $P_{i}$ are $t$-maximal (by Lemma 3.2). This is a contradiction.

$(2) \Rightarrow(1)$. By [16, Theorem 23.3], it suffices to show that every prime $*-$ ideal of $R$ is $*$-invertible. If $R$ satisfies ACC on $*$-invertible $*$-ideals, then by Lemma 2.2 , every $*$-invertible $*$-ideal is a $*$-product of $*$-coatoms. Thus by Lemma 2.3 , every $*$-invertible $*$-ideal is a $*$-product of prime $*$-ideals. Hence it follows from hypothesis and Proposition 2.4 that every $*$-maximal ideal of $R$ has height-one, that is, every prime $*$-ideal is $*$-maximal. Therefore again by hypothesis, every prime $*$-ideal of $R$ is $*$-invertible.

$(1) \Rightarrow(5)$. Let $I$ be a nonzero ideal of $R$. Then $\left(I I^{-1}\right)_{*}=R$ by $(1)$, and hence $I$ is $t$-invertible and $I_{*}=I_{t}$ by Lemma 2.2. Thus $R$ is a Krull domain and $*=t$.

$(5) \Rightarrow(1)$. This is clear.

$(4) \Rightarrow(6)$. This follows from Theorem 3.7 .

$(6) \Rightarrow(2)$. Let $M$ be a $*$-maximal ideal of $R$. Choose a nonzero $a_{1} \in M$. If $a_{1} R=M$, then $M$ is $*$-invertible; so assume $a_{1} R \subsetneq M$. Choose another $a_{2} \in M \backslash a_{1} R$. If $\left(a_{1}, a_{2}\right) R=M$, then $M$ is $*$-invertible because $R$ is $*$-Prüfer. If $\left(a_{1}, a_{2}\right) R \subsetneq M$, choose an $a_{3} \in M \backslash\left(a_{1}, a_{2}\right) R$. Repeating this process, we have $a_{1} R \subseteq\left(a_{1}, a_{2}\right) R \subseteq \cdots \subseteq M$. Since $R$ is *-Prüfer, each nonzero finitely generated ideal is $*$-invertible, and hence there is an integer $n$ such that $M=\left(a_{1}, \ldots, a_{n}\right) R$. Thus $M$ is $*$-invertible.

$(5) \Rightarrow(7)$. This follows from the fact that an integral domain $R$ is a Krull domain if and only if $R$ is a $t$-Prüfer and $t$-factorable domain ([8, Theorem 9$]$ ).

$(7) \Rightarrow(1)$. Mutatis mutandis, the proof is analogous to that of $[8$, Theorem $9]$.

$(5) \Rightarrow(8)$. This follows from the fact that an integral domain $R$ is a Krull domain if and only if $R$ is a unique $t$-factorable domain ([8, Theorem 12]).

$(8) \Rightarrow(5)$. This follows from [10, Theorem 3.2 and Corollary 3.3].

Acknowledgement. The authors would like to thank the referee for his/her many helpful suggestions. 


\section{References}

[1] D. D. Anderson, $\pi$-domains, overrings, and divisorial ideals, Glasgow Math. J. 19 (1978), no. 2, 199-203.

[2] _ Star-operation induced by overrings, Comm. Algebra 16 (1988), no. 12, 25352553.

[3] D. D. Anderson and D. F. Anderson, Generalized GCD-domains, Comment. Math. Univ. St. Paul. 28 (1980), no. 2, 215-221.

[4] D. D. Anderson and S. Chun, Commutative rings with finitely generated monoids of fractional ideals, J. Algebra 320 (2008), no. 7, 3006-3021.

[5] D. D. Anderson, T. Dumitrescu, and M. Zafrullah, Quasi-Schreier domains II, Comm. Algebra 35 (2007), no. 7, 2096-2104.

[6] D. D. Anderson, J. L. Mott, and J. Park, Finitely generated monoids of fractional ideals, Comm. Algebra 21 (1993), no. 2, 615-634.

[7] D. D. Anderson and M. Zafrullah, Integral domains in which nonzero locally principal ideals are invertible, Comm. Algebra 39 (2011), no. 3, 933-941.

[8] D. F. Anderson, H. Kim, and J. Park, Factorable domains, Comm. Algebra 30 (2002), no. 9, 4113-4120.

[9] A. Bouvier and M. Zafrullah, On some class groups of an integral domain, Bull. Soc. Math. Grèce (N.S.) 29 (1988), 45-59.

[10] G. W. Chang and J. Park, Star-invertible ideals of integral domains, Boll. Unione Mat. Ital. Sez. B Artic. Ric. Mat. (8) 6 (2003), no. 1, 141-150.

[11] T. Dumitrescu and R. Moldovan, Quasi-Schreier domains, Math. Rep. (Bucur.) 5(55) (2003), no. 2, 121-126.

[12] T. Dumitrescu and M. Zafrullah, t-Schreier domains, Comm. Algebra 39 (2011), no. 3, 808-818.

[13] S. El Baghdadi, M. Fontana, and G. Picozza, Semistar Dedekind domains, J. Pure Appl. Algebra 193 (2004), no. 1-3, 27-60.

[14] S. Gabelli, On domains with ACC on invertible ideals, Atti Accad. Naz. Lincei Rend. Cl. Sci. Fis. Mat. Natur. (8) 82 (1988), no. 3, 419-422.

[15] R. Gilmer, Multiplicative Ideal Theory, Queen's University, Kingston, Ontario, 1992.

[16] F. Halter-Koch, Ideal Systems: An Introduction to Multiplicative Ideal Theory, Marcel Dekker, New York, 1998.

[17] B. G. Kang, On the converse of a well-known fact about Krull domains, J. Algebra 124 (1989), no. 2, 284-299.

[18] H. Kim, M. O. Kim, and Y. S. Park, Some characterizations of Krull monoids, Algebra Colloq. 14 (2007), no. 3, 469-477.

[19] H. Kim and Y. S. Park, Some characterizations of Krull domains, J. Pure Appl. Algebra 208 (2007), no. 1, 339-344.

[20] R. B. Treger, Rings with a free semigroup of invertible ideals, Mat. Sb. (N.S.) 89(131) (1972), 100-109.

[21] M. Zafrullah, Putting t-invertibility to use, in Non-Noetherian Commutative Ring Theory, 429-457, Math. Appl., 520, Kluwer Acad. Publ., Dordrecht, 2000.

[22] _ t-invertibility and Bazzoni-like statements, J. Pure Appl. Algebra 214 (2010), no. $5,654-657$.

Gyu Whan Chang

Department of Mathematics

UNIVERSITY OF INCHEON

INCHEON 406-772, KoREA

E-mail address: whan@incheon.ac.kr 
HWANKOO KIM

Department of Information SECURITy

Hoseo University

Asan 336-795, Korea

E-mail address: hkkim@hoseo.edu 